\title{
Percutaneous cardiopulmonary support to treat suspected venous air embolism with cardiac arrest during open eye surgery
}

- a case report-

\author{
Seokyung Shin ${ }^{1,2}$, Bokyung Nam ${ }^{1}$, Sarah Soh ${ }^{1,2}$, and Bon-Nyeo Koo ${ }^{1,2}$ \\ ${ }^{1}$ Department of Anesthesiology and Pain Medicine, ${ }^{2}$ Anesthesia and Pain Research Institute, Yonsei University College of Medicine, \\ Yonsei University Health System, Seoul, Korea
}

We report a case of possible venous air embolism (VAE) during trans pars plana vitrectomy with air-fluid exchange of the vitreous cavity. Shortly after initiation of air-fluid exchange, decreases in end-tidal $\mathrm{CO}_{2}$, oxygen saturation, and blood pressure were observed. The patient rapidly progressed to cardiac arrest unresponsive to cardiopulmonary resuscitation, and recovered after the application of percutaneous cardiopulmonary support. Prompt termination of air infusion is needed when VAE is suspected during air-fluid exchange, and extracorporeal life support should be considered in fatal cases. Although the incidence is rare the possibility of VAE during ophthalmic surgery clearly exists, and therefore awareness and vigilant monitoring seem critical. (Korean J Anesthesiol 2014; 67: 350-353)

Key Words: Air embolism, Ophthalmologic surgical procedures.

Venous air embolism (VAE) is known to be associated with various types of surgical procedures, and while many cases may go unrecognized or have only subclinical effects, it is nevertheless a potentially life-threatening event [1]. A few cases of air embolism during ophthalmic surgery using air-fluid exchange have been recently reported, with some resulting in fatal outcomes [2-6]. Although ophthalmic surgery is considered to be a low risk procedure for air embolism, awareness regarding the possibility of such an event is needed. We report a case of possible air embolism during open eye surgery in a patient with choroidal melanoma, which led to cardiovascular arrest that was treated with percutaneous cardiopulmonary support (PCPS). The patient of the present case has reviewed the case report and provided written permission for the authors to publish the report.

Received: December 30, 2013. Revised: 1st, February 4, 2014; 2nd, February 17, 2014. Accepted: February 18, 2014.

Corresponding author: Bon-Nyeo Koo, M.D., Ph.D., Department of Anesthesiology and Pain Medicine, Anesthesia and Pain Research Institute, Yonsei University College of Medicine, 50, Yonsei-ro, Seodaemun-gu, Seoul 120-749, Korea. Tel: 82-2-2228-2420, Fax: 82-2-312-7185, E-mail: koobn@yuhs.ac

(c) This is an open-access article distributed under the terms of the Creative Commons Attribution Non-Commercial License (http:// creativecommons.org/licenses/by-nc/3.0/), which permits unrestricted non-commercial use, distribution, and reproduction in any medium, provided the original work is properly cited. 


\section{Case Report}

A 42-year-old male patient (American Society of Anesthesiologists physical status II) diagnosed with choroidal melanoma was scheduled for elective three-port trans pars plana vitrectomy (TPPV) and brachytherapy. Other than a past history of pulmonary tuberculosis, the patient had no other underlying diseases or abnormal preoperative findings. After induction of general anesthesia, the patient was mechanically ventilated with an oxygen/air mixture at an $\mathrm{FiO}_{2}$ of 0.5 to maintain normocapnia. A 20-gauge infusion cannula, light source and vitreous cutter were transclerally inserted, and serous retinal detachment of the macula and choroidal melanoma situated at the nasal side of the optic disc were observed. Following tumor removal with the vitreous cutter, air-fluid exchange was initiated at a pressure of $35 \mathrm{mmHg}$ through the infusion cannula. A sharp decrease in end tidal $\mathrm{CO}_{2}\left(\mathrm{ETCO}_{2}\right)$ from 36 to $11 \mathrm{mmHg}$ and a drop in saturation from 100 to $90 \%$ were seen just minutes after air-fluid exchange was started. This was quickly followed by a decrease in systolic blood pressure from 100 to $50 \mathrm{mmHg}$ with premature ventricular complexes observed on electrocardiogram (ECG). Air-fluid exchange was immediately stopped, and the intravenous line was checked for possible disconnection or accidental air entrapment. The patient's vital signs rapidly deteriorated and progressed to cardiac arrest with ventricular fibrillation, which was unresponsive to defibrillation and cardiopulmonary resuscitation (CPR). Tracheal tube suction revealed copious amounts of pink frothy sputum, reflecting pulmonary edema. After approximately 87 minutes of unsuccessful CPR, central venous access was secured and PCPS was applied in a venoarterial mode by cannulating the common femoral artery and vein. After a percutaneous femoral arterial cannula (DLP, Medtronic Inc.,
Minneapolis, MN, USA) and femoral long venous cannula (DLP, Medtronic Inc.) were inserted, PCPS was initiated at a flow rate of $4.0 \mathrm{~L} / \mathrm{min}$, using the Capiox emergency bypass system (EBS, Terumo Corp., Tokyo, Japan). The patient's cardiac rhythm returned six minutes after application of PCPS, and an immediate bedside transthoracic echocardiogram (TTE) showed severe hypokinesia of the anterior wall with an ejection fraction (EF) of $30 \%$. Chest X-ray showed left pneumothorax, diffuse subcutaneous emphysema in both upper thoracic walls, rib fractures, and pulmonary edema. Sinus tachycardia and nonspecific ST and T wave abnormalities were seen on ECG. A postoperative coronary angiography revealed normal coronary arteries without any occlusive findings.

The patient was admitted to the intensive care unit and maintained on PCPS for five days, and extubated on post-operative day (POD) \#7. He demonstrated signs of short-term memory impairment and quadriparesis, and a brain magnetic resonance imaging (MRI) scan performed on POD \#12 revealed a multifocal subacute infarct along the cortical gray matter (Fig. 1A) and suspected air bubbles in the left temporal lobe (Fig. 1B). Followup TTE done on POD \#25 showed regional wall motion abnormality in the left anterior descending coronary artery territory with improved left ventricular function $(\mathrm{EF}=52 \%)$. Transesophageal echocardiography (TEE) revealed a grade II patent foramen ovale (PFO) without any intracardiac thrombi. The patient was able to ambulate independently after two months, with only mild memory impairment remaining.

\section{Discussion}

Although rare, there have been previous reports of air embolism during open eye surgery using air-fluid exchange [2-6].

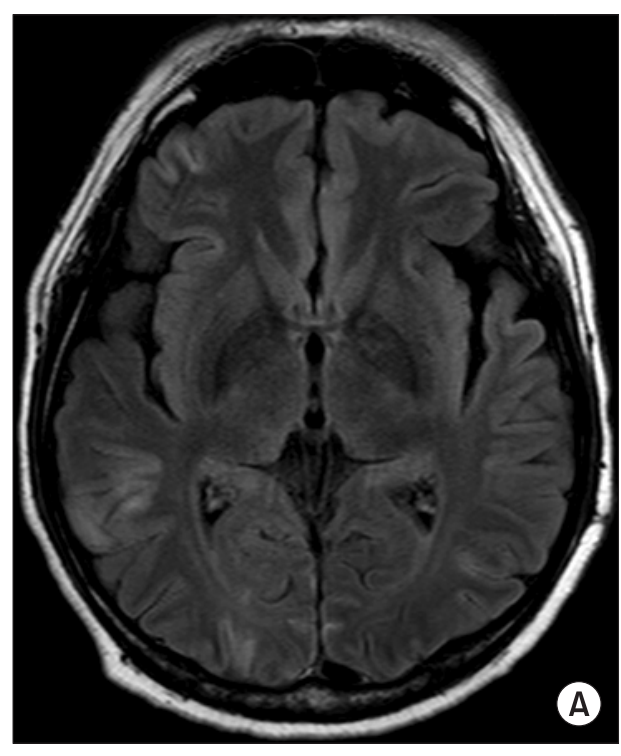

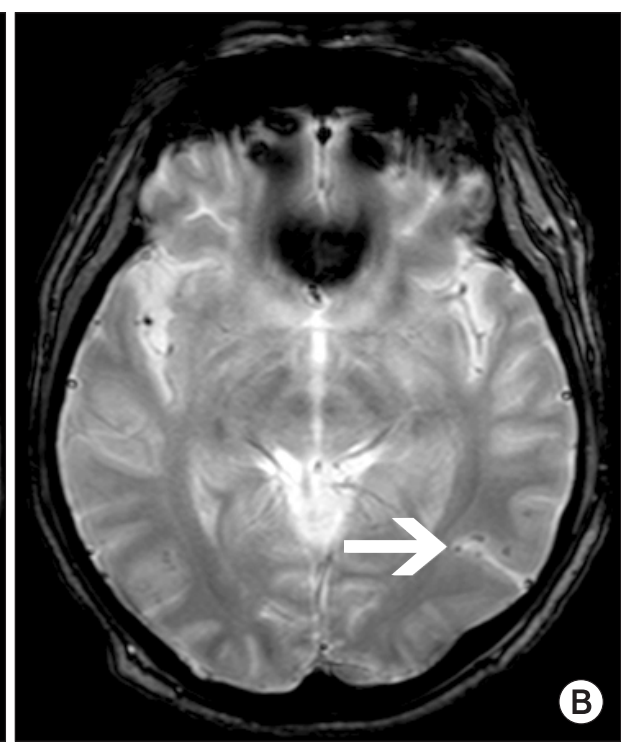

B
Fig. 1. (A) Brain MRI fluid-attenuated inversion recovery images taken 12 days after the event show multifocal bihemispheric lesions with a gyriform pattern in the bilateral cerebral cortices suggesting subacute infarcts. (B) A T2weighted gradient-echo MRI image taken 12 days after the event. Suspected air emboli are observed in the left temporal lobe (arrow). 
Concurrent ocular air-fluid exchange and VAE has been recently acknowledged as a potentially fatal event that all vitreoretinal surgeons should be aware of [7]. The initiating event in the case discussed here seems to be entrapment of air through abnormal venous access formed at the choroidal or vortex veins during removal of the tumor mass. The choroidal veins coalesce and drain into the vortex veins, which are then drained into the ophthalmic veins, and exit via the cavernous sinus, ultimately reaching the jugular veins. A large air embolism can create an air-lock within the right ventricle, and resulting secondary pulmonary hypertension may lead to reduced cardiac output and systemic hypotension [3].

Air-fluid exchange is performed after vitrectomy to close retinal tears, by replacing intraocular fluid from the posterior segment of the globe with air. As air enters the eye, fluid in the vitreous cavity is removed either passively or with active suction [8]. The air flow against atmospheric pressure when using $40 \mathrm{mmHg}$ infusion pressure through a 25 -gauge cannula is known to be approximately $350 \mathrm{ml}$ per minute [7], and this can be expected to be greater with a larger cannula and higher infusion pressure. The present case is similar to two previous case reports in which VAE occurred during vitrectomy for the removal of an intraocular foreign body [4]. A wound to an intraocular vein during the removal of the melanoma may have provided the entry site for air bubbles during air-fluid exchange. In addition, seconday retinal ischemia from serous retinal detachment is known to be a possible cause of retinal neovascularization associated with choroidal melanoma [9]. This may have rendered the patient in this case more susceptible to air entrapment due to increased blood flow in the retina.

The present case is further complicated by the fact that the patient was found to have a PFO upon TEE. Among the few previous reports of VAE during ophthalmic surgery, two cases resulted in patient death $[4,6]$, and one of these cases occurred in a patient with a PFO [4]. That patient developed myocardial infarction and multiple organ failure, and died four weeks after the event. The severity of the present case also seems to be related to the pre-existing PFO, which may have caused paradoxical air embolism (PAE) combined with VAE. The brain MRI findings were characteristic of cerebral embolism, showing patterns of restricted diffusion similar to those that had been observed in a previous report [10]. The multifocal ischemic areas were not mainly localized to the watershed zones, suggesting that the period of cardiac arrest may not have been entirely responsible for the neurologic complications. However, it is unclear whether PAE occurred initially through the PFO or was secondarily caused by the increased right ventricular pressure during chest compression. Compared to most cases, in which the events resolved after a short period of time without any complications, the patient in the present case showed rapid and severe cardio- vascular collapse that was unresponsive to CPR even after the prompt termination of air infusion. From this perspective, systemic arterial air embolism via the PFO as the triggering event seems a possible explanation of the present case. On the other hand, despite its low sensitivity for VAE detection, ECG changes are known to be seen early in the course of an event only with rapid entrainment of air [1]. ST and T changes followed by supraventricular and ventricular tachyarrythmias are typically observed in humans, as was also the case with the present patient. Air entrapment at an unintended high speed may have caused a relatively large amount of air to enter the patient's circulation in a very short period of time.

When VAE is suspected during air-fluid exchange, prompt termination of air infusion should be done in order to prevent further air entrainment. Nitrous oxide should be discontinued and $100 \%$ oxygen should be applied to maximize patient oxygenation and reduce embolus volume [1]. While CPR with defibrillation and chest compression has been demonstrated for massive VAE with cardiac arrest [11], the closed-chest massage itself is thought to force air out of the pulmonary outflow tract, thus improving forward blood flow [1]. The closed-chest massage has even been shown to be equally beneficial when compared to left lateral positioning and intracardiac air aspiration in animal studies [12]. When traditional methods of CPR are not effective and cardiovascular collapse is persistent, the initiation of PCPS should be considered. The most frequently reported indication for PCPS is cardiac arrest refractory to CPR, and prompt decision-making by the attending physician and a welltrained, available staff of cardiothoracic surgeons, perfusionists, and nurses are required to accomplish effective treatment [13]. Although a cardiac arrest period of over 30 minutes has been suggested as a relative contraindication to PCPS [13], the present case shows that the inititation of PCPS after a more extensive period of refractory cardiac arrest may still be life-saving with minimal sequelae.

TEE is currently the most sensitive diagnostic method for detecting air embolism [1], and real-time TEE during air-fluid exchange would have provided valuable insight into the actual cause of the initial event in the present case. The presence of intracardiac air following air-fluid exchange would have explained the assumed VAE, and the possible PAE leading to neurologic complications. Precordial Doppler and transcranial Doppler ultrasound are also useful monitors, both with the advantage of being highly sensitive among the noninvasive monitors [1]. The existing reports of VAE during ophthalmic surgery are all limited in that the aforementioned diagnostic modalities were unavailable at the time of the event, and therefore the suggested possible causes are at best speculative. Because we also had not been able to observe the presence of air bubbles during the initial event, we had planned to use TEE monitoring when the 
patient received a re-operation. Unfortunately, a second operation within two months was strongly discouraged by the department of neurology due to the high risk of worsening neurologic sequelae, and the patient's eye could not be salvaged at that point thus rendering TPPV meaningless.

Although the exact cause and incidence of air embolism dur- ing ophthalmic surgery are unknown, the possibility of such an event clearly exists. Vigilant monitoring with increased awareness on the part of the anesthesiologist seems most important in preventing fatal outcomes, while the application of extracorporeal life support modalities should also be considered in severe cases when circumstances permit.

\section{References}

1. Mirski MA, Lele AV, Fitzsimmons L, Toung TJ. Diagnosis and treatment of vascular air embolism. Anesthesiology 2007; $106:$ 164-77.

2. Ledowski T, Kiese F, Jeglin S, Scholz J. Possible air embolism during eye surgery. Anesth Analg 2005; 100: 1651-2.

3. Ruest P, Aroichane M, Cordahi G, Bureau N. Possible venous air embolism during open eye surgery in a child. Can J Anaesth 2007; 54: 840-4.

4. Lim LT, Somerville GM, Walker JD. Venous air embolism during air/fluid exchange: a potentially fatal complication. Arch Ophthalmol 2010; 128: 1618-9.

5. Kanbay A, Hasanoğlu HC, Karalezli A, Aykun G, Yülek F. Forgotten but an important risk factor for pulmonary embolism: ophthalmic surgery. Tuberk Toraks 2011; 59: 101-2.

6. Dermigny F, Daelman F, Guinot PG, Hubert V, Jezraoui P, Thomas F, et al. Fatal air embolism during open eye surgery. Ann Fr Anesth Reanim 2008; 27: 840-2.

7. Morris RE, Sapp MR, Oltmanns MH, Kuhn F. Presumed air by vitrectomy embolisation (PAVE) a potentially fatal syndrome. Br J Ophthalmol 2014; 98: 765-8.

8. Peyman GA, Schulman JA. Posterior segment techniques. In: Intravitreal surgery : principles and practice. 2nd ed. Norwalk, Appleton \& Lange. 1994, pp 281-356.

9. Lee J, Logani S, Lakosha H, Schroeder RP, Simpson R, Jampol LM. Preretinal neovascularisation associated with choroidal melanoma. Br J Ophthalmol 2001; 85: 1309-12.

10. Caulfield AF, Lansberg MG, Marks MP, Albers GW, Wijman CA. MRI characteristics of cerebral air embolism from a venous source. Neurology 2006; 66: 945-6.

11. Ericsson JA, Gottlieb JD, Sweet RB. Closed-chest cardiac massage in the treatment of venous air embolism. N Engl J Med 1964; $270: 1353-4$.

12. Takeoka M, Sakai A, Ueda G, Ge RL, Panos RJ, Taniguchi S. Influence of hypoxia and pulmonary air embolism on lung injury in perfused rat lungs. Respiration 1996; 63: 346-51.

13. Kurusz M, Zwischenberger JB. Percutaneous cardiopulmonary bypass for cardiac emergencies. Perfusion 2002; 17: 269-77. 